\title{
Subsegmental Pulmonary Embolism: Value of Thoracic Ultrasound for Diagnosis and Follow-Up
}

\author{
Claudio Mastruzzo ${ }^{1}$, Guido Perracchio ${ }^{2}$, Grazia Poidomani ${ }^{1}$, \\ Marcello Romano ${ }^{2}$, Nunzio Crimi ${ }^{1}$ and Carlo Vancheri ${ }^{1}$
}

\begin{abstract}
Timely diagnosis of pulmonary embolism (PE) is crucial because prompt appropriate management can decrease mortality and morbidity. However, the diagnosis of $\mathrm{PE}$ is often a challenge because of aspecific clinical presentation and the lack of a single non-invasive diagnostic test sufficiently sensitive for the diagnosis in all suspected cases. The present report describes a 37-year-old woman in whom diagnosis of recurrent subsegmental PE was achieved through subtle integration of imaging techniques including thoracic ultrasound (TUS) that was demonstrated to be of valuable usefulness. We think that TUS may represent an adjunctive technique for diagnosing PE.
\end{abstract}

Key words: computed tomography, pulmonary embolism, thoracic ultrasound

(Inter Med 47: 1415-1417, 2008)

(DOI: 10.2169/internalmedicine.47.1085)

\section{Introduction}

Pulmonary embolism (PE) is a common cardiopulmonary illness representing the most underdiagnosed condition among internal diseases and is responsible for a large number of preventable deaths (1). Timely diagnosis of PE is crucial because prompt appropriate management can decrease mortality and morbidity, but is often confounded by aspecific clinical presentation. Moreover, there is virtually no single non-invasive diagnostic test which is sufficiently sensitive for the diagnosis in all suspected cases. The present report describes a 37-year-old woman admitted to hospital with chest pain and fever. Thoracic ultrasound (TUS) was demonstrated to be of valuable usefulness to achieve the diagnosis of recurrent PE. We think that TUS may represent a reliable adjunctive technique for diagnosing $\mathrm{PE}$.

\section{Case Report}

A 37-year-old woman was admitted to the hospital with fever and right hemithorax pain. There were not other symptoms. She had been generally well, had no knowledge of previous significant diseases and reported having taken oral contraceptives for 1 year. At physical examination her temperature was $38.5^{\circ} \mathrm{C}$, the pulse rate was 96 beats per minute, the respiratory rate was $18 / \mathrm{min}$, and the blood pressure was $120 / 70 \mathrm{mmHg}$. The lungs were clear and physical examination was normal. $\mathrm{PaO}_{2}$ was $80 \mathrm{mmHg}$ and $\mathrm{PaCO}_{2} 41 \mathrm{mmHg}$ breathing room air. A thorough cardiovascular exam disclosed no cardiac abnormalities. There was no calf pain or tenderness. Electrocardiogram, echocardiogram and lung function tests were normal. A chest radiography and a helical computed-tomography (CT) scan of the chest did not reveal any abnormalities with the exception of a modest right pleural effusion. Laboratory tests revealed an increased white blood cell count (WBCc) and enhanced erythrocyte sedimentation rate (ESR). Later on, she complained again of a novel acute chest pain, but this time in the basal left hemithorax. New laboratory tests showed an increased ddimer. A pulmonary ventilation/perfusion scintigraphy detected bilateral perfusion defects and was interpreted as an intermediate probability scan resulting not diagnostic while a CT pulmonary angiography (CTPA) did not show any remarkable findings and confirmed the presence of a right pleural effusion that appeared slightly reduced. Duplex ultra-

${ }^{1}$ Department of Internal and Specialistic Medicine, Section of Respiratory Diseases, University of Catania, Catania, Italy and ${ }^{2}$ Division of Geriatric Medicine, Tomaselli Hospital, Catania, Italy

Received for publication March 5, 2008; Accepted for publication May 8, 2008

Correspondence to Dr. Claudio Mastruzzo, claudiomastruzzo@yahoo.it 


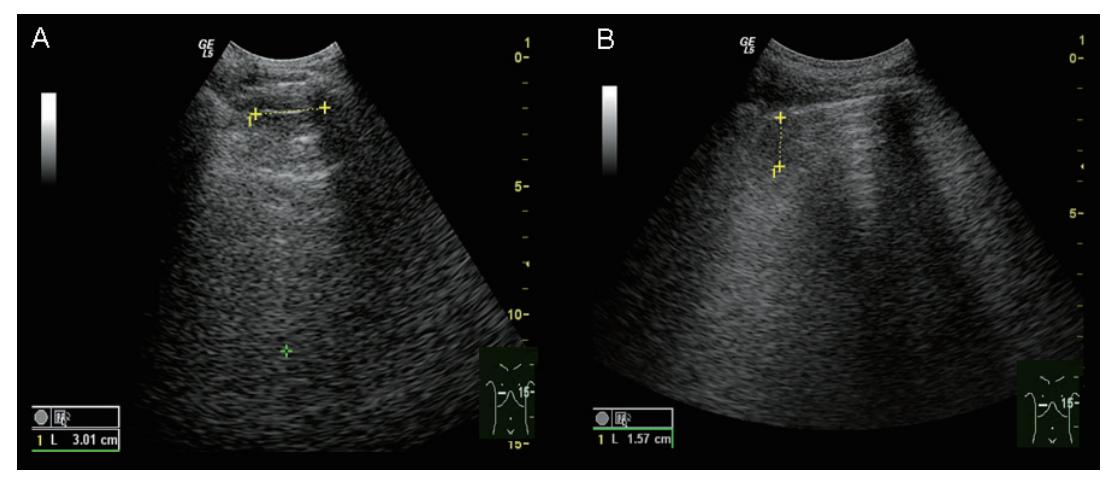

Figure 1. A: ultrasound image showing a small rounded subpleural right posterior basal lesion (dotted yellow line). B: ultrasound image showing halving of the same lesion (dotted yellow line) 20 days later.

sonography of legs revealed no deep vein thrombosis. At this point we planned a pulmonary angiography but the patient refused it. Thus, we performed a thoracic ultrasound (TUS) which showed 3 bilateral (one left and two right) rounded well-demarcated pleura-based hypoechoic areas. Sonographic features (location, number, size and form) were suggestive of thromboembolic lesions (Fig. 1A). The patient started enoxaparin and warfarin. The enoxaparin was discontinued when the INR reached a therapeutic level. No evidence of other causes of thromboembolic disease was found. Work-up for a coagulation defect could not be performed at this point because of anticoagulant therapy. A few days later, chest pain and fever disappeared and d-dimer, as well as other laboratory values were decreased down to normal values. Following serial TUS demonstrated progressive size reduction of hypoechoic areas. 20 days after starting anticoagulant therapy, TUS showed complete resolution of two subpleural lesion and halving of one (Fig. 1B), while a CTPA done at this time was negative for vascular and parenchimal lesions. The patient remained in good condition and she is well 6 months after discharge.

\section{Discussion}

$\mathrm{PE}$ is a frequently underdiagnosed and undertreated disease (1). Unfortunately, as a result of non-specific clinical symptoms and signs, attaining a timely diagnosis is fraught with challenge. Pulmonary angiography, considered as the gold standard for the diagnosis of PE, is an invasive procedure associated with some risk and is underutilized in traditional diagnostic strategies. Moreover, although much attention has been paid to optimizing the diagnosis of acute PE, there is uncertainty about the accuracy of non-invasive imaging techniques. In fact, in most patients with suspected $\mathrm{PE}$, ventilation-perfusion scan results are not definitive and non-diagnostic. CT pulmonary angiography (CTPA) is increasingly preferred as the reference method for the noninvasive diagnosis of PE, but the diagnostic yield of CTPA in subsegmental PE is still debated $(2,3)$.

In the present patient, the clinical features at the beginning, including fever, increased $\mathrm{WBCc}$ and ESR were sug- gestive for an infectious process, delaying the correct diagnosis. However, the recurrence of migrant chest pain and recognition of increased d-dimer prompted the diagnosis of recurrent $\mathrm{PE}$ which was finally achieved mainly through findings obtained by TUS.

The value of TUS in the diagnosis of acute PE has been reported in previous studies (4-6). Sonomorphology of thromboembolic lesions is typical and specific features include localization, number, size and form of hypoecogen areas. A number of criteria can be used for sonomorphologic differential diagnosis between PE and peripheral pulmonary lesions of other origin. Notably, in most of the studies, TUS showed an excellent resolution of thromboembolic lesions in the subpleural region, demonstrating an overall sensitivity comparable with that obtained by CTPA $(4,6)$. Of interest, in our patient, repeat CTPA did not demonstrate the occurrence of PE that was detected by TUS instead. Also the V/Q scan was not diagnostic. Thus, the present report stresses important concerns regarding actually recommended noninvasive imaging techniques for PE detection. Particularly, in some instances, the diagnostic accuracy of CTPA in the diagnosis of PE is not satisfactory and peripheral subsegmental thromboembolic lesions may be overlooked by CTPA. Several studies have demonstrated that performance of CTPA showed variability in sensitivity with the poorest sensitivity results for subsegmental subpleural emboli (7-11), which are, on the other hand, those better revealed by TUS. Although the clinical importance of peripheral emboli is still unclear (12), we think that the early diagnosis of subsegmental PE is essential. Follow-up studies of untreated patients with initially negative test results found PE in patients with normal CT or other imaging procedures with a frequency up to $28 \%(9,10,13-15)$. These findings demonstrate that false negative diagnoses may commonly occur and highlight the limitations of all the currently available imaging investigations for the detection of subsegmental pulmonary emboli due to the fall of their diagnostic capability from the central to the peripheral pulmonary arteries. In those patients with initially unrecognized PE, the integration of the currently utilized imaging procedures with TUS may increase sensibility in detection of subsegmental embolism. 
Indeed, recurrence of PE may easily occur in untreated patients with subsegmental PE which may represent a harbinger of major PE and even patients with minor symptoms are at risk of potentially life threatening thromboembolism requiring rigorous treatment in order to reduce mortality due to PE. In fact, it is recognized that the majority of deaths due to PE occur in patients who are not treated because of the lack of appropriate diagnosis. We think that, in selected patients, the presence of clinical suspicion and increased ddimer together with pleura-based hypoechoic areas at TUS may strongly suggest a diagnosis of subsegmental embolism even in the absence of remarkable findings in the currently available imaging investigations.

Moreover, thromboembolism is a highly dynamic event with rapidly changing features with respect to radiologic findings $(16,17)$. The resolution rate of thromboemboli in patients with acute pulmonary embolism may range from a few days to several months and pulmonary infarctions may be reabsorbed within a few hours or days. Currently imaging investigations, such as CTPA and V/Q scan, often requires time-consuming organization prior to the investigation and their non ready availability in the right time window may affect their sensitivity representing a critical pitfall in the diagnosis of acute PE. Importantly, in most clinical settings, TUS represents a widely available procedure which allows bedside examination of patients without delay for appropriate treatment avoiding the time factor of spontaneous lysis. TUS may also be useful as routine re-imaging procedure to follow the clinical course of disease during and after cessation of anticoagulant therapy.

In conclusion, clinical practice underlines the fact that the cornerstone for the diagnosis of PE is clinical suspicion followed by a subtle clinical algorithm including physical evaluation, d-dimer test, CTPA, V/Q scintigraphy, echocardiography and leg vein duplex sonography. Nevertheless, a timely diagnosis of PE is still a challenge and justifies the application of all available diagnostic procedures useful to achieve the diagnosis of acute PE. TUS may represent a reliable adjunctive technique for diagnosing PE especially in some circumstances, such as subsegmental subpleural embolism. Moreover, TUS is a re-imaging technique of choice in order to evaluate the resolution rate of pulmonary infarction and investigate symptomatic recurrence of PE.

\section{References}

1. Goldhaber SZ. Pulmonary embolism. Lancet 363: 1295-1305, 2004.

2. Fedullo PF, Tapson VF. The evaluation of suspected pulmonary embolism. N Engl J Med 349: 1247-1256, 2003.

3. Musset D, Parent F, Meyer G, et al. Diagnostic strategy for patients with suspected pulmonary embolism: a prospective multicentre outcome study. Lancet 360: 1914-1920, 2002.

4. Reissig A, Heyne JP, Kroegel C. Sonography of lung and pleura in pulmonary embolism: sonomorphologic characterization and comparison with spiral CT scanning. Chest 120: 1977-1983, 2001.

5. Mathis G, Blank W, Reissig A, et al. Thoracic ultrasound for diagnosing pulmonary embolism: a prospective multicenter study of 352 patients. Chest 128: 1531-1538, 2005.

6. Reissig A, Kroegel C. Transthoracic ultrasound of lung and pleura in the diagnosis of pulmonary embolism. A novel non-invasive bedside approach. Respiration 70: 441-452, 2003.

7. Perrier A, Howarth N, Didier D, et al. Performance of helical computed tomography in unselected outpatients with suspected pulmonary embolism. Ann Intern Med 135: 88-97, 2001.

8. Hogg K, Brown G, Dunning J, et al. Diagnosis of pulmonary embolism with CT pulmonary angiography: a systematic review. Emerg Med J 23: 172-178, 2006.

9. Goodman LR, Lipchik RJ, Kuzo RS, Liu Y, MoAuliffe TL, O'Brien DJ. Subsequent pulmonary embolism: risk after a negative helical CT pulmonary angiogram-prospective comparison with scintigraphy. Radiology 215: 535-542, 2000.

10. Jiménez D, Gómez M, Herrero R, et al. Thromboembolic events in patients after a negative computed tomography pulmonary angiogram: a retrospective study of 165 patients. Arch Bronconeumol 42: 344-348, 2006.

11. Stein PD, Fowler SE, Goodman LR, et al. Multidetector computed tomography for acute pulmonary embolism. N Engl J Med 354: 2317-2327, 2006.

12. Le Gal G, Righini M, Parent F, van Strijen M, Couturaud F. Diagnosis and management of subsegmental pulmonary embolism. J Thromb Haemost 4: 724-731, 2006.

13. Garg K, Sieler H, Welsh $\mathrm{CH}$, Johnston RJ, Russ PD. Clinical validity of helical CT being interpreted as negative for pulmonary embolism: implications for patient treatment. Am J Roentgenol 172: 1627-1631, 1999.

14. Ferretti GR, Bosson JL, Buffaz PD, et al. Acute pulmonary embolism: role of helical CT in 164 patients with intermediate probability at ventilation-perfusion scintigraphy and normal results at duplex US of the legs. Radiology 205 (2): 453-458, 1997.

15. Henry JW, Relyea B, Stein PD. Continuing risk of thromboemboli among patients with normal pulmonary angiograms. Chest 107: 1375-1378, 1995.

16. Kroegel C, Reissig A. Principle mechanisms underlying venous thromboembolism: epidemiology, risk factors, pathophysiology and pathogenesis. Respiration 70: 7-21, 2003.

17. Nijkeuter M, Hovens MMC, Davidson BL, Huisman MV. Resolution of thromboemboli in patients with acute pulmonary embolism: a systematic review. Chest 129: 192-197, 2006.

(C) 2008 The Japanese Society of Internal Medicine http://www.naika.or.jp/imindex.html 